\title{
CHANGE IN THE WARM ABSORBER IN MR 2251-178
}

\author{
C. OTANI ${ }^{1}$, T. KII ${ }^{2}, \&$ K. MIYA ${ }^{2}$ \\ 1 The Institute of Physical and Chemical Research (RIKEN) \\ 2 The Institute of Space and Astronautical Science (ISAS)
}

\begin{abstract}
We report the results of the search for the change in the warm absorber of the quasar MR 2251-178 with $A S C A$. We detected its change along with the X-ray intensity change. In the bright phase in 1993, its Xray spectra showed the absorption edge features by OVII and OvIII edges. In contrast, in the medium intensity (in 1996), the absorption apparently increased and the edge energy was shifted to the lower energy. These results are well explained by the ionization change of the same absorber. The timescale of the change requires moderately high density and the location to be nearer to the center than the Narrow Line Region. We briefly discuss the stability of the warm absorber.
\end{abstract}

\section{Results}

Six $A S C A$ observations of MR 2251-178 were performed during 2 months in 1993 and four during 6 months in 1996. The source was in the bright phase in 1993 and in the medium phase in 1996. The absorption feature of the mean spectrum in 1993 was well reproduced by the combination of OviI and OvIII edges in the quasar's rest frame $(z=0.064)$. The significant change of the feature from 1993 is detected in 1996. The edge energy was shifted from 0.74 to $0.67 \mathrm{keV}$, and the bulk absorption increased (Figure). Both spectra are well reproduced by a single warm absorber model with the ionization parameter of $\log \xi=1.35$ and 0.96 in 1993 and 1996, respectively. The total column density was almost constant $\left(\log N_{\mathrm{W}}=21.6-21.7\right)$. This means that the observed change of the absorption feature is explained by the change of the single warm absorber. The warm absorber fits of the individual spectra show that $\xi$ correlates with the flux and that $N_{\mathrm{W}}$ stays almost constant (see Figure again). 

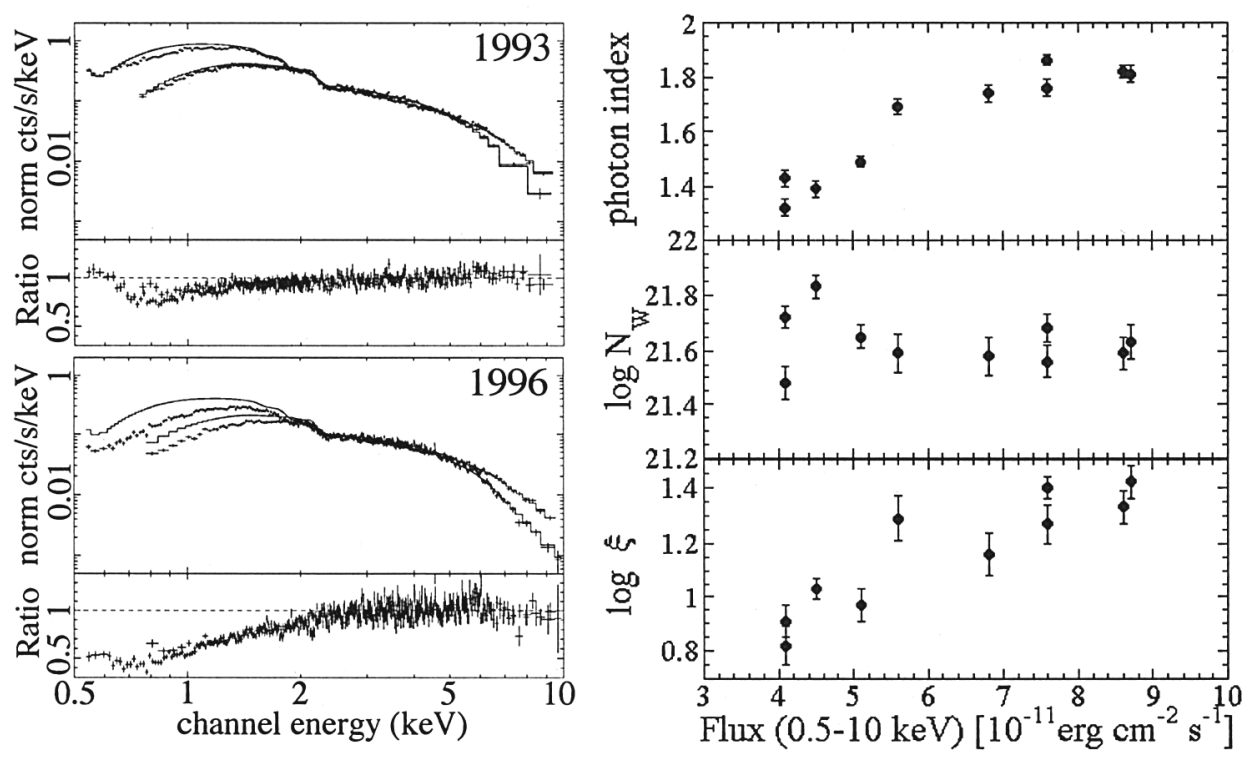

Figures. (left) Mean spectra and the residuals from the power-law model determined above $3 \mathrm{keV}$ in 1993 (upper) and 1996 (lower). (right) The correlation of the incident flux in $0.5-10 \mathrm{keV}$ with $\Gamma, N_{\mathrm{W}}$, and $\xi$ in the individual observations.

\section{Discussion}

The smooth correlation between the flux and the ionization parameter implies that the recombination timescale in the absorber is shorter than about months to a year. This gives the lower limit of the density to be $\sim 10^{4-5} \mathrm{~cm}^{-3}$ and the distance from the center to be smaller than $\sim 10 \mathrm{pc}$. Since the quasar's narrow line region (NLR) is expect to locate around 1-10 $\mathrm{kpc}$, the warm matter should locate closer to the center than NLR.

We explored the thermal stability of the warm absorber in terms of the "two-phase" model (Krolik, McKee, Tarter 1981). Adopting the observed flux-index $(\Gamma)$ relation, we calculated the $\Xi-T$ equilibrium curves for $\Gamma=1.1-1.9$ using Cloudy (Ferland 1996). We found that, though the curves changes and the ionization state of the absorber in 1993 moves as $\Gamma$ changes, its track seems keep stable. Although this result is still preliminary, such a behavior may explain the stable presence of the warm absorber in MR 2251-178.

\section{References}

Ferland G.J. 1996, 'Hazy, a Brief Introduction to Cloudy', Univ. of Kentucky, Dept. of Physics and Astronomy

Krolik J.H., McKee C.F., Tarter C.B. 1981, ApJ 371, 541 